\title{
Asymptomatic high grade osteosarcoma of proximal tibia: a case report
}

\begin{abstract}
Osteosarcomas are primary malignant tumors that are characterized by the production of osteoid or immature bone. Osteosarcoma is a deadly form of musculoskeletal cancer that most commonly causes patients to die from pulmonary metastatic disease and which has a 5-year survival rate of $15-20 \%$. A 19 -year-old female patient came to the hospital for mild right knee pain following history of fall one day back. Before this she had no complaints in her knees. The patient was investigated for the same which revealed high grade osteosarcoma of proximal tibia. She underwent preoperative chemotherapy, followed by limb-saving operation. This case report underlines the significance of proper evaluation and high index of suspicion in adolescent ager group about malignant bony growths.
\end{abstract}

Volume 8 Issue 5 - 2017

\author{
Manish Diwakar \\ Shankus Hospital,Ahmedabad, India
}

Correspondence: Manish Diwakar, Shankus Hospital, A 203 Shukan Residency near Vandemataram City, New S G Road, Gota, Ahmedabad, India, Tel 9099989950;

Email manish.diwakar@gmail.com

Received: April 02, 2017 | Published: July 21, 2017

\section{Introduction}

Osteosarcomas are uncommon malignant bone tumors, which are characterized by the production of osteoid or immature bone by the malignant cells. The incidence has a bimodal distribution with peaks in early adolescence and in elderly.

In children, osteosarcomas account for approximately 3 percent of childhood cancers overall. The peak incidence is during the adolescent growth spurt. In adults, osteosarcomas are often considered as secondary neoplasms. About one-third of osteosarcomas in adults arise in a background of Paget's diseases of bone or previous cancer. Several risk factors of osteosarcoma in adults are recognized, such as Paget's disease, inherited conditions, benign bone tumor, and prior irradiation or chemotherapy.

Almost all osteosarcoma patients complain of symptoms related to their lesion at the first medical visit. Physical findings are regional pain, palpable mass, restricted movement of joint and others. A previous study about complaints of malignant bone tumors reported that $85 \%$ patients had pain related strain, $21 \%$ had pain at night, $47 \%$ had the onset of symptoms related to minor trauma and $39 \%$ had a palpable mass.

Here we present a case of knee pain following trauma which turned out to be high grade osteosarcoma.

\section{Case report}

A 19-year-old female was referred to our hospital because of mild knee pain following trivial trauma one day back. She did not suffer any pain before this. A radiograph of her knee was taken. The result showed increased bone density in proximal tibia (sclerosis). However, no symptoms such as palpable mass, atrophy of muscle, fever and weight loss were found.

She did not have any medical history, without history of benign bone lesion, Paget's disease, inherited conditions, and prior irradiation or chemotherapy. She had no family history of malignancy. Her vital signs were within normal limits with a blood pressure of 122/84 $\mathrm{mmHg}$, a pulse of 84 beats per minute, a temperature $36.8^{\circ} \mathrm{C}$, and a room air oxygen saturation of $99 \%$. The weight was $54 \mathrm{~kg}$, height 155 . $\mathrm{cm}$, and body mass index 22.5 . The serum alkaline phosphatase level was not high (294U/l), and the levels of total protein, albumin, lactate dehydrogenase, blood urea nitrogen, sodium, potassium, chloride and $\mathrm{C}$-reactive protein were within normal limits. Liver and renal function was normal.
Her leg radiograph revealed sclerosis in the metaphysis of the proximal tibia, (Figure 1).

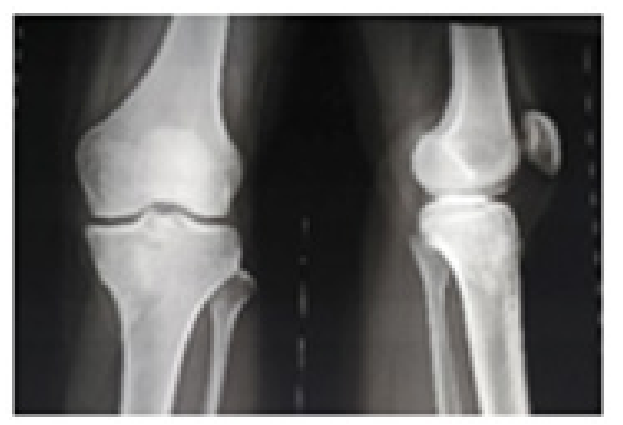

Figure I Radiograph of the left knee. There is a sclerotic lesion in the proximal tibia.

Magnetic resonance imaging (MRI) showed that a mass occupied the same part of the left tibia ( $6 \mathrm{~cm}$ and $3.5 \mathrm{~cm}$ and $3.0 \mathrm{~cm}$ in diameter) where sclerosis was seen on the radiograph, and that soft tissues around the lesion were edematous (Figure 2). Bone scintigraphy showed an abnormal uptake on the proximal part of the left tibia. Pulmonary CT was normal.

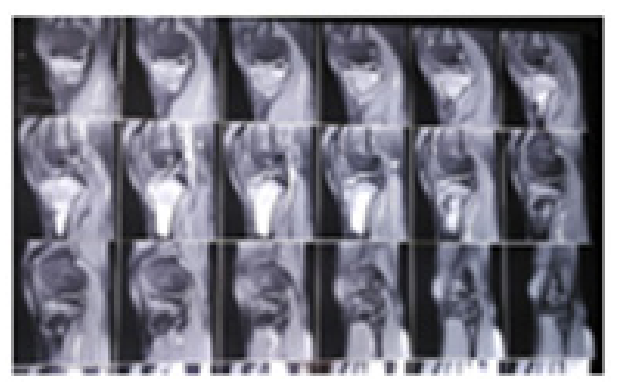

Figure $2 M R$ images of the left knee showing extent of growth and tissue oedema.

Biopsy was done, and the lesion was diagnosed as high grade osteosarcoma.

We started her on neoadjuvant chemotherapy, combination with cisplatin and adriamycin. We performed resection of the left upper tibia and arthroplasty with megaprosthesis (Figure 3). She was mobilized on next day but knee bending was not allowed. Stitches were removed 15 days postoperatively. After 4 weeks she was allowed knee bending with good return to daily activities. 

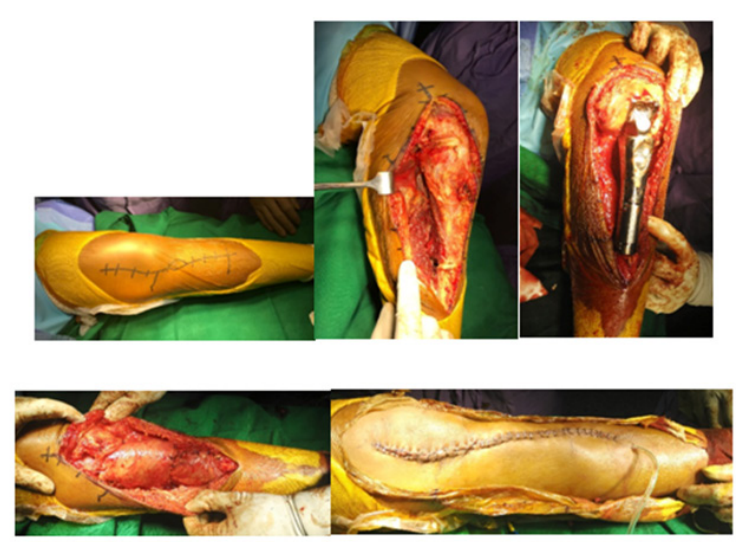

Figure 3 Intraoperative steps.
a. Incision
b. Dissection
c. Implant placement
d. Coverage with flap
e. closure

\section{Pathological findings}

A surgically resected specimen showed $5.0 \times 3 \times 3 \mathrm{~cm}$-sized tumor, located in the metaphysis of the left proximal tibia. The histological findings of this lesion mentioned absence of osteiod.

\section{Discussion}

Patients of osteosarcoma generally have some symptoms such as pain or palpable mass. ${ }^{1-3}$ Widhe (2000) reported initial symptoms and clinical features in osteosarcomas. ${ }^{1}$ According to the study, physical findings, at the first medical visit, of osteosarcomas are local tenderness
(93\%), palpable masses (39\%), and painful movement of joint (39\%), limp (31\%) and restricted movement of joint $(23 \%)$, atrophy of muscle $(5 \%)$ and fever (3\%). Systemic symptoms such as weight loss, malaise and dullness are uncommon. Thus, the diagnosis of osteosarcoma in adults is difficult. In our case, if she had not diagnosed by chance, she might be misdiagnosed leading to non-optimal treatment or be diagnosed by a pathological fracture, respiratory or other symptoms after she got worse or when she suffered pathological fracture.

\section{Conclusion}

Here we have presented a case of asymptomatic high grade osteosarcoma which was treated surgically with megaprosthesis resulting in good patient outcome and recovery.

Every cause of knee pain in adolescent age group should be properly investigated with high index of suspicion for malignant lesions to avoid chances of misdiagnosis or late diagnosis.

\section{Acknowledgments}

None.

\section{Conflicts of interest}

None.

\section{References}

1. Widhe $\mathrm{B}$, Widhe $\mathrm{T}$ Initial Symptoms and Clinical Features in Osteosarcoma and Ewing Sarcoma. J Bone Joint Surg Am. 2000;82(5):667-674.

2. Wang LL, Chintagumpala M, Gebhardt MC Osteosarcoma: Epidemiology, Pathogenesis, Clinical Presentation,Diagnosis, and Histology. 2014

3. Hornicek FJ Bone Sarcomas: Preoperative Evaluation, Histologic Classification, and Principles of Surgical Management. 2015. 